\title{
A ROBUST PASSIVE POWER-BASED CONTROL STRATEGY FOR THREE-PHASE VOLTAGE-SOURCE RECTIFIERS
}

\author{
Dimitri Jeltsema* Jacquelien M.A. Scherpen* \\ Elodie Hageman ** \\ * Delft Center for Systems and Control \\ Delft University of Technology \\ Mekelweg 2, 2628 CD Delft, The Netherlands. \\ j.m.a.scherpen@dcsc.tudelft.nl \\ ${ }^{* *}$ Ecole Centrale de Nantes \\ 1, Rue de la Noë, B.P. 92101 44321, \\ Nantes Cedex 3, France
}

\begin{abstract}
In this paper, robustness of the damping injection schemes for passivity based control (PBC) of the three-phase AC/DC voltage-source (Boost type) rectifier is studied. The idea is to extend the tuning strategy for $\mathrm{PBC}$ that is presented in (Jeltsema and Scherpen, 2004) for DC/DC converters to three-phase AC/DC rectifiers. In the DC/DC converter case this tuning strategy leads to a PBC scheme with parallel damping injection that is robust against load perturbations. Straightforward application of this scheme to the three-phase AC/DC Boost rectifier, however, does not immediately lead to such robust closed loop system. A closer analysis reveals that first a pre-compensation should be done so that the AC input stage connected to the DC output stage is converted into two DC input stages connected to the DC output stage. Then, the PBC parallel damping injection scheme can be applied resulting in a closed-loop system that is robust against load variations. Copyright (c) 2005 IFAC
\end{abstract}

Keywords: Passivity-Based Control, Brayton-Moser Equations, Power Converters, Nonlinear Control.

\section{INTRODUCTION}

In the last decade, passivity-based control (PBC) design for switched-mode power converters has become quite an active area in both the field of system and control theory and power electronics. One particular $\mathrm{PBC}$ technique is based on the classical Euler-Lagrange (EL) equations. The application of EL-based PBC design to single-switch DC/DC power converters was first proposed in (Sira-Ramírez et al., 1997; Ortega et al., 1998) and is generalized to larger networks, like the coupled-inductor Ćuk converter, and threephase rectifiers and inverters - see e.g. (Scherpen et al., 2003; Lee, 2004), and the references therein.

\footnotetext{
$\star$ This research is partly sponsored by the European Union Erasmus Program.
}

One of the major advantages of using the EL approach is that the physical structure (e.g., energy, dissipation and interconnection), including the nonlinear phenomena and features, is explicitly incorporated in the model, and thus in the corresponding PBC. This in contrast to conventional techniques that are mainly based on linearized dynamics and corresponding PID (Proportional-Integral-Derivative) or lead-lag control. Since many power converters are nonlinear non-minimum phase systems, controllers stemming from linear techniques are sometimes difficult to tune as to ensure robust performance, especially in the presence of large set point changes and disturbances that cause circuit operation to deviate from the nominal point of operation. Therefore, incorporating knowledge about the nonlinear dynamics in the controller design may be beneficial. 
However, a key issue for EL-based PBC that only recently obtained some attention is the selection and tuning of various control parameters. In (Jeltsema and Scherpen, 2004) we have presented some guidelines concerning the adjustments of the control parameters using modified versions of the stability theorems proposed in (Brayton and Moser, 1964). The theory is based on considering the so-called mixed-potential functions of the system which are closely related to the power in the system. Hence, the PBC scheme studied in (Jeltsema and Scherpen, 2004) is basically a powerbased PBC scheme. This scheme in combination with the theorems of (Brayton and Moser, 1964) justify the possibility to choose either a series or a parallel damping injection scheme, while in most EL-based PBC designs the location where to add the damping is mainly motivated by the form of the open-loop dissipation structure. ${ }^{1}$ Additionally, but not less important, a major advantage of the parallel damping injection PBC scheme in comparison with series damping injection is that it robustifies the closed-loop system since it does not require adaptive or integral extensions in case the load is unknown or varying, as is usually the case in a practical situation.

So far, the ideas in (Jeltsema and Scherpen, 2004) only concerned relatively simple DC/DC switchedmode converters (like the elementary Buck and Boost converters). In this paper, we study a more elaborate switched-mode converter that is widely used and studied in industry: the three-phase AC/DC voltage-source (Boost type) rectifier. As will become clear in the next sections, for the three-phase voltage-source rectifier the nice robustness properties of the parallel damping injection PBC strategy will be lost due to the structure at the AC-side of the system. However, comparing the structure of the system with the DC/DC Boost converter system results in a pre-compensation scheme that can be interpreted as a cancellation of the ACstructure in the system. Then, the ideas of (Jeltsema and Scherpen, 2004) can be applied straightforwardly, such that the parallel damping injection scheme results in a robust against load variation PBC scheme. A disadvantage is that in this case the system is not robust against variations in the input inductances due to the pre-compensation scheme. However, for applications the input inductances are often constant or only slowly varying. Thus, an adaptive mechanism to compensate for these variations is likely to be less difficult to implement and less costly than an adaptive mechanism that has to compensate for load variations.

The paper is structured as follows. Section 2 recapitulates some preliminaries and presents the model structure that we use in the sequel. Section 3 treats the full power-based $\mathrm{PBC}$ scheme for the three phase AC/DC Boost rectifier. Then, in Section 4 we propose the precompensation scheme which results in a system that

\footnotetext{
1 Usually, damping is added to those (error) states that do not
} contain any damping terms a priori. has two separate DC input stages that are connected to the DC output stage. Then we apply the parallel damping injection scheme. In Section 5 we present some practical considerations about the new control scheme, and in Section 6 we show the simulation results for a realistic closed loop AC/DC Boost rectifier. Finally, we end with some conclusions in Section 7.

\section{PRELIMINARIES: THE SWITCHED-MODE BRAYTON-MOSER EQUATIONS}

In (Brayton and Moser, 1964) it is shown that a large class of nonlinear electrical networks $\mathscr{N}$ can be described by a set of nonlinear first-order differential equations of the form (for ease of notation, we omit the functional arguments)

$$
\mathscr{N}:\left\{\begin{aligned}
-L \frac{\mathrm{d} i}{\mathrm{~d} t} & =\frac{\partial P}{\partial i} \\
C \frac{\mathrm{d} u}{\mathrm{~d} t} & =\frac{\partial P}{\partial u}
\end{aligned}\right.
$$

where the vectors $i$ and $u$ denote the currents and voltages associated to the set of independent linear inductors and capacitors, represented by the matrices $L$ and $C$, respectively. ${ }^{2}$ The topological relationships of the network are captured by a single scalar function $P$ - called the mixed-potential function. For networks without switches the mixed-potential function takes the general form:

$$
P=\underbrace{\int\left(u_{r}-\frac{1}{2} \gamma u\right) \mathrm{d} i}_{\text {content }}-\underbrace{\int\left(i_{g}+\frac{1}{2} \gamma^{T} i\right) \mathrm{d} u}_{\text {co-content }},
$$

where the vector functions $u_{r}=\hat{u}_{r}(i)$ and $i_{g}=\hat{i}_{g}(u)$ represent the characteristics of the current-controlled and voltage-controlled resistors and sources, respectively. The terms containing the matrix $\gamma$ can be considered as the power flows between the inductors and capacitors. This suggests that the interconnection between the inductors and capacitors can be viewed as ideal transformers with turns-ratio matrix $\gamma$. For more details on such interpretation, see e.g. (Stern, 1965). Note that $P$ is related to the total power of the circuit and that the dimension is that of power. Also note that the set of equations (1) is just an alternative way the represent Kirchhoff's voltage (KVL) and current (KCL) laws.

For DC networks that contain ideal switches $\sigma$, which take values in the discrete set $\{0,1\}$, the set of equations ( 1 ) is modified by replacing $P$ by a parameterized mixed-potential $P^{\sigma}$. For example, if a network contains a single switch $\sigma$, which is assumed to be $\mathrm{ON}$ $(\sigma=1)$ or OFF $(\sigma=0)$, it is easily seen that such parameterization is defined by

$$
P^{\sigma}=\sigma P^{1}+(1-\sigma) P^{0}
$$

\footnotetext{
2 The inductors and capacitors in this paper are assumed to be linear, but the framework also allows nonlinear inductors and capacitors.
} 
where $P^{1}$ (resp., $P^{0}$ ) represents the mixed-potential potential function for the network topology associated to the switch position $\sigma=1$ (resp. $\sigma=0$ ). Similar conditions hold for more general switching policies. For more details on the construction of such functions, the reader is referred to (Jeltsema and Scherpen, 2004).

\section{POWER-BASED PBC}

In (Jeltsema and Scherpen, 2004) we have studied the tuning of the parameters for a PBC scheme based on the mixed potential function for DC/DC converters. Some guidelines were presented concerning the adjustments of the control parameters based on modified versions of the stability theorems proposed in (Brayton and Moser, 1964). This resulted in a robust parallel damping injection scheme for DC/DC converters. In this section, we apply the technique suggested in (Jeltsema and Scherpen, 2004) to derive a power-based $\mathrm{PBC}$ scheme for the three-phase voltage source rectifier depicted in Figure 1, and study the robustness properties of the parallel damping strategy.

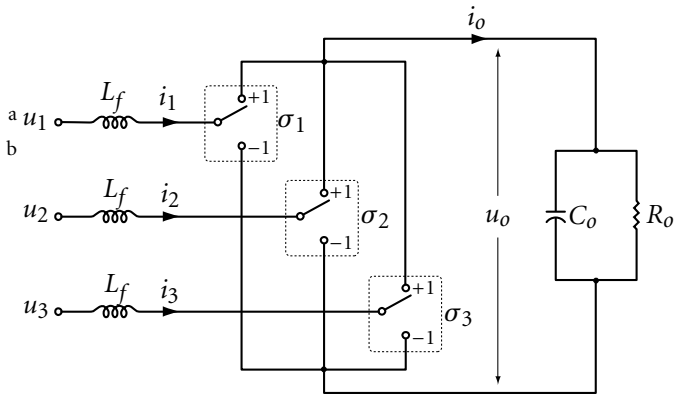

Fig. 1. Power stage of the three-phase AC/DC voltagesource rectifier.

Similar to EL-based PBC design, for the derivation of a power-based $\mathrm{PBC}$ we need to consider the rectifiers pulse-width modulated (PWM) dynamics, instead of the discrete switched-mode model. This means that, under the condition that the switching frequency is sufficiently high, we may replace the discrete switching functions $\sigma_{k} \in\{-1,1\}$, with $k=1,2,3$, by their corresponding duty-ratios $s_{k} \in[-1,1]$. Notice that $s_{k}$ may be considered as the average values of $\sigma$, or, adopting the interpretation of (Stern, 1965), $s_{k}$ are the modulated 'turns-ratios' of a bank of ideal interconnection transformers. Hence, we need to consider an averaged parameterized mixed-potential $P^{s}$, which for the rectifier of Figure 1 is constructed as follows. The first step in the power-based PBC design procedure of (Jeltsema and Scherpen, 2004) is the derivation of a set of average Brayton-Moser equations. Let $i_{k}$ denote the average input inductor currents, $u_{o}$ denote the average output capacitor voltage, and let the source voltages

$$
u_{k}:\left\{\begin{array}{l}
u_{1}=U_{f} \cos (\omega t) \\
u_{2}=U_{f} \cos (\omega t-2 \pi / 3), \\
u_{2}=U_{f} \cos (\omega t+2 \pi / 3),
\end{array}\right.
$$

with $U_{f}$ the peak amplitude and $\omega$ the radial frequency.
Since $\hat{u}_{r}\left(i_{k}\right)=u_{k}, \hat{i}_{g}\left(u_{o}\right)=R_{o}^{-1} u_{o}$, and

$$
\hat{\gamma}(s)=-\frac{1}{2} \operatorname{diag}\left(s_{1}, s_{2}, s_{3}\right),
$$

the averaged parameterized mixed-potential is readily found as

$$
\begin{aligned}
P^{s}= & \sum_{k=1}^{3} \int\left(-u_{k}+\frac{1}{4} s_{k} u_{o}\right) \mathrm{d} i_{k} \\
& -\int\left(\frac{1}{R_{o}} u_{o}-\frac{1}{4} \sum_{k=1}^{3} s_{k} i_{k}\right) \mathrm{d} u_{o} \\
= & -\sum_{k=1}^{3} u_{k} i_{k}+\frac{1}{2} u_{o} \sum_{k=1}^{3} s_{k} i_{k}-\frac{1}{2 R_{o}} u_{o}^{2},
\end{aligned}
$$

yielding the differential equations

$$
\mathscr{N}:\left\{\begin{aligned}
-L_{f} \frac{\mathrm{d} i_{k}}{\mathrm{~d} t}=\frac{\partial P^{s}}{\partial i_{k}}=-u_{k}+\frac{1}{2} s_{k} u_{o} \\
C_{o} \frac{\mathrm{d} u_{o}}{\mathrm{~d} t}=\frac{\partial P^{s}}{\partial u_{o}}=\frac{1}{2} \sum_{k=1}^{3} s_{k} i_{k}-\frac{1}{R_{o}} u_{o},
\end{aligned}\right.
$$

for $k=1,2,3$.

Remark: Note that in the cases where the duty ratio functions take the extreme values $s=(-1,-1,-1)$, $s=(-1,-1,1), \ldots, s=(1,1,1)$, for $s=\left(s_{1}, s_{2}, s_{3}\right)$, one recovers the corresponding parameterized potentials $P^{\sigma}$ — which are eight in total.

The rectifier must fulfill two objectives: first, the output capacitor voltage should maintain a constant desired value $u_{o}^{*}=U_{o}$; second, the rectifier should operate with an unity power factor, which means that the input inductor currents $i_{k}$ should be in phase with the source voltages $u_{k}$, i.e., $i_{k}=I_{f} U_{f}^{-1} u_{k}$, where $I_{f}$ denotes the amplitude of the average inductor currents.

Consider then the error variables $e_{k}=i_{k}-\xi_{k}$, and $e_{o}=$ $u_{o}-\xi_{o}$, where $\xi_{(\cdot)}$ are some auxiliary variables (i.e., desired trajectories for $i_{k}$ and $u_{o}$ ) to be defined later. Following the power-based PBC methodology (Jeltsema and Scherpen, 2004), we want to shape the closed-loop mixed-potential to a desired mixed-potential:

$$
P_{\mathrm{d}}^{s}=\frac{1}{2} e_{o} \sum_{k=1}^{3} s_{k} e_{k}-\frac{1}{2 R_{o}} e_{o}^{2}-\frac{1}{2 R_{p}} e_{o}^{2},
$$

where $R_{p}$ is the injected 'virtual' resistor in parallel with the output capacitor $C_{o}$. This yields the desired closed-loop stabilization error dynamics

$$
\mathscr{N}_{\mathrm{d}}:\left\{\begin{aligned}
-L_{f} \frac{\mathrm{d} e_{k}}{\mathrm{~d} t} & =\frac{1}{2} s_{k} e_{o} \\
C_{o} \frac{\mathrm{d} e_{o}}{\mathrm{~d} t} & =\frac{1}{2} \sum_{k=1}^{3} s_{k} e_{k}-\left(\frac{1}{R_{o}}+\frac{1}{R_{p}}\right) e_{o}
\end{aligned}\right.
$$

Based on the theorems in (Jeltsema and Scherpen, 2004), we can deduce from the latter set of equations that the control objective is achieved if we set

$$
\frac{1}{R_{p}}=\frac{1}{1-\delta} \sqrt{\frac{C_{o}}{L_{k}}}-\frac{1}{R_{o}},
$$

where $0<\delta<1$ is a fine-tuning parameter. The associated (implicit) controller dynamics can be obtained by 
taking the respective gradients of the controller mixedpotential

$$
\begin{aligned}
P_{\mathrm{c}}^{s}=-\sum_{k=1}^{3} u_{k} \xi_{k}+ & \frac{1}{2} \xi_{o} \sum_{k=1}^{3} s_{k} \xi_{k} \\
& -\frac{1}{2 R_{o}} \xi_{o}^{2}-\frac{1}{2 R_{p}}\left(u_{o}-\xi_{o}\right)^{2},
\end{aligned}
$$

i.e.,

$$
\mathscr{N}_{\mathrm{c}}:\left\{\begin{aligned}
-L_{f} \frac{\mathrm{d} \xi_{k}}{\mathrm{~d} t} & =-u_{k}+\frac{1}{2} s_{k} \xi_{o} \\
C_{o} \frac{\mathrm{d} \xi_{o}}{\mathrm{~d} t} & =\frac{1}{2} \sum_{k=1}^{3} s_{k} \xi_{k}-\frac{1}{R_{o}} \xi_{o}+\frac{1}{R_{p}}\left(u_{o}-\xi_{o}\right),
\end{aligned}\right.
$$

Basically, the rationale behind the design of a parallel damping PBC consists in making a copy of the model (5), add damping in the error of the capacitor voltage, and then solve for the controls $s_{k}$. Since the last step involves a partial system inversion it is necessary to ensure that the associated state(s) are minimum phase, i.e., the zero-dynamics of the part of the system to be inverted are stable. It can be shown that the only feasible solution is to indirectly control the output voltage via regulation of the input inductor currents by setting $\xi_{k}=i_{k}^{*}$, where $i_{k}^{*}=I_{k} U_{f}^{-1} u_{k}$ denote the desired equilibrium values for the input inductor currents. Hence, by substituting $\xi_{k}=i_{k}^{*}$ in the implicit controller dynamics resulting from the respective gradients of (9) and solving for $s_{k}$, yields the explicit control laws:

$$
s_{k}=\frac{2}{\xi_{o}}\left(u_{k}-L_{f} \frac{\mathrm{d} i_{k}^{*}}{\mathrm{~d} t}\right),
$$

where the auxiliary variable $\xi_{o}$, with $\xi_{o}(0)>0$, is the solution of the nonlinear differential equation

$$
C_{o} \frac{\mathrm{d} \xi_{o}}{\mathrm{~d} t}=\frac{1}{2} \sum_{k=1}^{3} s_{k} i_{k}^{*}-\frac{1}{R_{o}} \xi_{o}+\frac{1}{R_{p}}\left(u_{o}-\xi_{o}\right) .
$$

Convergence of the errors $e_{k} \rightarrow 0, e_{o} \rightarrow 0$, as well as $\xi_{o} \rightarrow U_{o}$ can be proved by substituting (11) into (7), and by simultaneously evaluating (12).

The unavoidable practical problem that arises is when the load resistor is not exactly known. This is most easily shown as follows. Suppose the load is composed of a known nominal value $R_{o}$ and a bounded uncertainty $\Delta R_{o}$, such that $R_{o}+\Delta R_{o}>0$. In that case, the input current amplitude, say $I_{f}$, is directly dependent on the unknown load resistor, i.e., $I_{f}\left(R_{o}^{\prime}\right)=U_{o}^{2} /\left(R_{o}^{\prime} U_{f}\right)$, where $R_{o}^{\prime} \triangleq R_{o}+\Delta R_{o}$. On the other hand, since $\Delta R_{o}$ is unknown, the nominal set-point used for the indirect regulation scheme is $I_{a}=U_{o}^{2} /\left(R_{o} U_{f}\right)$, which means that (11) can be rewritten

$$
s_{k}=\frac{2}{\xi_{o}}\left(u_{k}-\frac{L_{f} I_{a}}{U_{f}} \dot{u}_{k}\right),
$$

where $\dot{u}_{k}=-\omega U_{f} \sin (\omega t \pm \cdots)$. Hence, if $\Delta R_{o} \neq 0$, the amplitude of the error, say $E_{k}$, (and thus $e_{k}$ !) will not converge to zero as desired, but instead $E_{k} \rightarrow I_{f}-I_{a} \neq 0$. A similar discussion holds for $e_{o}$, resulting in the fact that $u_{o}^{*} \neq U_{o}$.
Since it is physically impossible to manipulate $\Delta R_{o}$, the nice features of parallel damping injection, as for the class of converters in (Jeltsema and Scherpen, 2004), are lost. This means for the present scenario that the only solution to robustify the closed-loop is to extend the PBC with an adaptive mechanism (or outer-loop PI controller (Kugi, 2001)) to estimate the actual load resistor $R_{o}+\Delta R_{o}$. However, this results in a computationally more expensive controller. Additionally, the passivity properties of the closed-loop will then be lost. In order to make a more transparent analysis of the problem, it is beneficial to consider the Park transformation, which is done in the next section.

\section{PRE-COMPENSATION SCHEME}

Since the three-phase source is balanced, we apply a Park transformation to the open-loop dynamics (5). This means that, instead of the three-phase input inductor currents $i_{k}$ and duty ratios $s_{k}$, we consider their associated direct and quadrature values, $i_{d}, i_{q}, s_{d}$, and $s_{q}$, respectively, in the so-called $d q$-frame, which rotates at a constant angular frequency $\omega$. For details, see e.g., (Leonhard, 1985). The resulting open-loop dynamics in the $d q$-frame read:

$$
\mathscr{N}:\left\{\begin{aligned}
L_{f} \frac{\mathrm{d} i_{\mathrm{d}}}{\mathrm{d} t} & =\sqrt{\frac{2}{3}} U_{f}-\omega L_{f} i_{q}-\frac{1}{2} s_{d} u_{o} \\
L_{f} \frac{\mathrm{d} i_{q}}{\mathrm{~d} t} & =\omega L_{f} i_{d}-\frac{1}{2} s_{q} u_{o} \\
C_{o} \frac{\mathrm{d} u_{o}}{\mathrm{~d} t} & =\frac{1}{2} s_{d} i_{d}+\frac{1}{2} s_{q} i_{q}-\frac{1}{R_{o}} u_{o} .
\end{aligned}\right.
$$

In the $d q$-frame, the control objective can be reformulated as follows. Find controls $s_{d}$ and $s_{q}$ such that for all unknown $R_{o}^{\prime}>0$ :

C.1 The output capacitor voltage converges to its desired equilibrium value $u_{o}^{*}=U_{o}$;

C.2 The power factor of the rectifier asymptotically converges to one, i.e., $i_{d}^{*}=I_{d}$ and $i_{q}^{*}=0$, where $I_{d}\left(R_{o}^{\prime}\right)=U_{o}^{2} /\left(2 R_{o}^{\prime} U_{d}\right)$, with $U_{d} \triangleq \sqrt{2 / 3} U_{f}$.

Thus, the control objective reduces to a set-point regulation problem. Via the Park transformation, the cross terms $-\omega L_{f} i_{q}$ and $+\omega L_{f} i_{d}$ are directly related to the derivative terms $L_{f} \dot{\xi}_{k}$ in (11), which are causing the sensitivity to unmodeled changes in the load resistor.

To overcome this problem, suppose that we are able to precisely cancel the cross terms $-\omega L_{f} i_{q}$ and $+\omega L_{f} i_{d}$. Theoretically, this could be accomplished by selecting controls of the form

$$
s_{d}=-\frac{\omega L_{f} i_{q}}{u_{o}}+2 \overline{s_{d}}, \quad s_{q}=\frac{\omega L_{f} i_{d}}{u_{o}}+2 \overline{s_{q}},
$$

for all $u_{o}(0)>0$, and where $\overline{s_{d}}$ and $\overline{s_{q}}$ are some new control inputs. We come back to the practical issues later on. Hence, substitution of (15) into (14) yields for the resulting 'pre-compensated' dynamics 


$$
\mathscr{N}_{\mathrm{pc}}:\left\{\begin{array}{l}
L_{f} \frac{\mathrm{d} i_{\mathrm{d}}}{\mathrm{d} t}=U_{d}-\overline{s_{d}} u_{o}, L_{f} \frac{\mathrm{d} i_{q}}{\mathrm{~d} t}=-\overline{s_{q}} u_{o} \\
C_{o} \frac{\mathrm{d} u_{o}}{\mathrm{~d} t}=\overline{s_{d}} i_{d}+\overline{s_{q}} i_{q}-\frac{1}{R_{o}} u_{o} .
\end{array}\right.
$$

It is recognized that we now have two separate DC input stages which are connected to the DC output stage as shown in Figure 2. In a similar fashion as before, we can define a desired mixed-potential in terms of the error variables $e_{d}=i_{d}-\xi_{d}, e_{q}=i_{q}-\xi_{q}$, and $e_{o}=u_{o}-\xi_{o}$, and try again a parallel damping injection scheme, i.e.,

$$
P_{\mathrm{d}}^{s}=\left(\overline{s_{d}} e_{d}+\overline{s_{q}} e_{q}\right) e_{o}-\frac{1}{2 R_{o}} e_{o}^{2}-\frac{1}{2 R_{p}} e_{o}^{2} .
$$

The error stabilization dynamics are

$$
\mathscr{N}_{\mathrm{d}}:\left\{\begin{aligned}
-L_{f} \frac{\mathrm{d} e_{\mathrm{d}}}{\mathrm{d} t} & =\frac{\partial P_{\mathrm{d}}^{\mathrm{s}}}{\partial e_{d}}=\overline{s_{d}} e_{o} \\
-L_{f} \frac{\mathrm{d} e_{q}}{\mathrm{~d} t} & =\frac{\partial P_{\mathrm{d}}^{s}}{\partial e_{q}}=\overline{s_{q}} e_{o} \\
C_{o} \frac{\mathrm{d} e_{o}}{\mathrm{~d} t} & =\frac{\partial P_{\mathrm{d}}^{s}}{\partial e_{o}}=\overline{s_{d}} e_{d}+\overline{s_{q}} e_{q}-\left(\frac{1}{R_{o}}+\frac{1}{R_{p}}\right) e_{o} .
\end{aligned}\right.
$$

Again by invoking the theorems in (Jeltsema and Scherpen, 2004) the control objective is achieved if we set

$$
\frac{1}{R_{p}}=\frac{\max \left\{s_{d}, s_{q}\right\}}{1-\delta} \sqrt{\frac{C_{o}}{L_{f}}}-\frac{1}{R_{o}},
$$

where $0<\delta<1$ is a fine-tuning parameter, whereas the associated (implicit) controller dynamics are obtained by taking the respective gradients of the controller mixed-potential

$$
\begin{aligned}
P_{\mathrm{c}}^{s}=-U_{d} \xi_{d}+ & \left(\bar{s}_{d} \xi_{d}+\bar{s}_{q} \xi_{q}\right) \xi_{o} \\
& -\frac{1}{2 R_{o}} \xi_{o}^{2}-\frac{1}{2 R_{p}}\left(u_{o}-\xi_{o}\right)^{2},
\end{aligned}
$$

i.e.,

$$
\mathscr{N}_{\mathrm{c}}:\left\{\begin{aligned}
-L_{f} \frac{\mathrm{d} \xi_{\mathrm{d}}}{\mathrm{d} t} & =-U_{d}+\bar{s}_{d} \xi_{o},-L_{f} \frac{\mathrm{d} \xi_{q}}{\mathrm{~d} t}=\overline{s_{q}} \xi_{o} \\
C_{o} \frac{\mathrm{d} \xi_{o}}{\mathrm{~d} t} & =\bar{s}_{d} \xi_{d}+\bar{s}_{q} \xi_{q}-\frac{1}{R_{o}} \xi_{o}+\frac{1}{R_{p}}\left(u_{o}-\xi_{o}\right) .
\end{aligned}\right.
$$

By C.1 and C.2, the explicit PBC controls $\overline{s_{d}}$ and $\overline{s_{q}}$ are found by letting $\xi_{d}=I_{a}$ and $\xi_{q}=0$, i.e.,

$$
\overline{s_{d}}=\frac{U_{d}}{\xi_{o}}, \quad \overline{s_{q}}=0,
$$

where the auxiliary variable $\xi_{o}$, with $\xi_{o}(0)>0$, now is the solution of

$$
C_{o} \frac{\mathrm{d} \xi_{o}}{\mathrm{~d} t}=\frac{U_{d}}{\xi_{o}} I_{d}-\frac{1}{R_{o}} \xi_{o}+\frac{1}{R_{p}}\left(u_{o}-\xi_{o}\right) .
$$

The rectifier dynamics (14), with $\Delta R_{o} \neq 0$, in closedloop with the inner-loop pre-compensator (15) and the outer-loop parallel damping injection $\mathrm{PBC}$ scheme (18) - (19) then take the form:

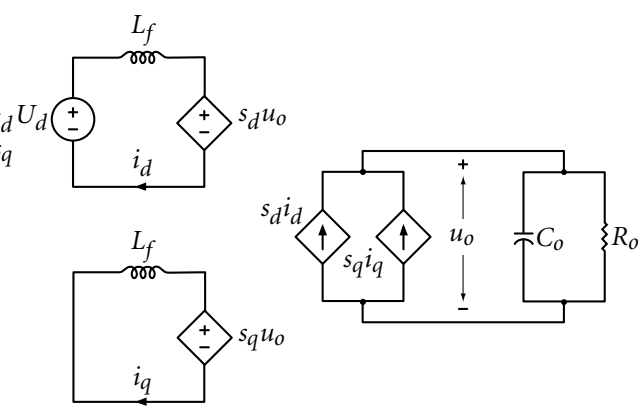

Fig. 2. Equivalent network representation of the precompensated rectifier.

$$
\mathscr{N}_{\mathrm{cl}}:\left\{\begin{aligned}
L_{f} \frac{\mathrm{d} i_{\mathrm{d}}}{\mathrm{d} t}=U_{d}-\frac{U_{d}}{\xi_{o}} u_{o}, \quad L_{f} \frac{\mathrm{d} i_{q}}{\mathrm{~d} t}=0 \\
C_{o} \frac{\mathrm{d} u_{o}}{\mathrm{~d} t}=\frac{U_{d}}{\xi_{o}} i_{d}-\frac{1}{R_{o}^{\prime}} u_{o} \\
C_{o} \frac{\mathrm{d} \xi_{o}}{\mathrm{~d} t}=\frac{U_{d}}{\xi_{o}} I_{a}-\left(\frac{1}{R_{o}}+\frac{1}{R_{p}}\right) \xi_{o}+\frac{1}{R_{p}} u_{o} .
\end{aligned}\right.
$$

Interestingly, we note that control objective C.2 is always satisfied since the direct and quadrature current equations are decoupled and the time-variation of $i_{q}$ is always zero. Regarding C.1, it is easily observed that at equilibrium $u_{o}=\xi_{o}$, which implies that $u_{o}=u_{o}^{*}=U_{o}$ and necessarily $i_{d}=i_{d}^{*}=I_{d}$, for time goes to infinity. Hence, we have shown that, under the condition that we can find controls (15), the robustness ideas presented in (Jeltsema and Scherpen, 2004) still remain valid.

\section{PRACTICAL ISSUES}

In the previous section, we have shown that, under the condition that we can find controls (15), the robustness ideas presented in (Jeltsema and Scherpen, 2004) still remain valid. Of course, one practical assumption is that $i_{d}, i_{q}$ and $u_{o}$ are available for measurement (i.e., full-state knowledge is necessary). Additionally, in order to fully cancel the cross terms in the $d q$-frame, the quantity $\omega L_{f}$ needs to be known exactly. Another problem is the fact that the pre-compensation scheme involves a division by $u_{o}$, which is usually equal to zero at start-up. Since in a 'real-world' rectifier the input inductances and $\omega$ are usually constant, or at most (very) slowly varying, we could extend the controller with an adaptive mechanism that estimates the value of of the term $\omega L_{f}$ during the start-up of the rectifier. In this way, we have shifted the problem of a constant adaptation of the load resistor, as it is usually done, to only a temporary adaptation of the inductance values and the value the angular frequency. This is an additional computational effort, however, in practice this is often far less costly than the computational effort that would be necessary for changes in the load resistor. Concerning the division problem, it is easily shown that if we replace $u_{o}$ in (15) by its desired equilibrium value $U_{o}$, which is strictly positive by definition, we obtain the same equilibrium results as concluded at 

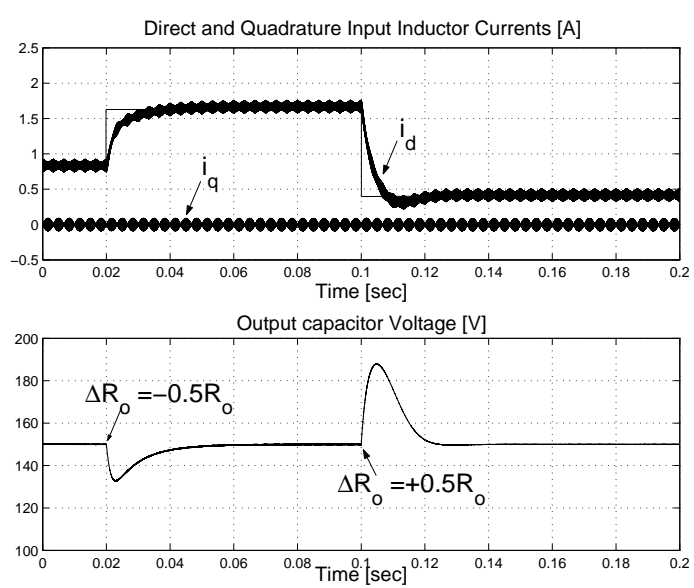

Fig. 3. Closed-loop response to step changes in the load resistor. At $t=0.02 \mathrm{sec}$ a load perturbation of $\Delta R_{o}=-\frac{1}{2} R_{o}$ is applied, and at $t=0.1 \sec \Delta R_{o}=$ $+\frac{1}{2} R_{o}$.

the end of the previous section. Summarizing, we have the following statement.

Proposition: Consider the three-phase rectifier dynamics (14), in closed-loop with the controller

$$
s_{d}=-\frac{\omega L_{f} i_{q}}{U_{o}}+\frac{U_{d}}{\xi_{o}}, \quad s_{q}=\frac{\omega L_{f} i_{d}}{U_{o}},
$$

where $\xi_{o}$, with $\xi_{o}(0)>0$, is the solution of (19). If the injected parallel resistor $R_{p}$ satisfies (16), then the trajectories of the closed-loop system converge to their desired equilibrium values in a non-oscillatory way, regardless of $\Delta R_{o}>-R_{o}$.

\section{NUMERICAL RESULTS}

For demonstrating the validity of the theoretical derivations and developments, a simulation study using Matlab/Simulink is performed for the switched (i.e., not averaged) current and voltage states. Limiters are used to ensure the duty-ratios of the actual switch control signals do not exceed their physical boundaries. The design parameters of the rectifier are set as follows: $U_{f}=100 \mathrm{~V}, L_{f}=10 \mathrm{mH}, C_{o}=47 \mu \mathrm{F}, R_{o}=220 \Omega$, $F_{s}=20 \mathrm{kHz}$. Suppose it is desired that the rectifier operates at a constant DC output voltage $U_{o}=150 \mathrm{~V}$. The results are depicted in Figure 3. The top figure shows the responses of the switched-mode input inductor currents $i_{d}(t)$ and $i_{q}(t)$, and the bottom figure shows the response of the switched-mode output capacitor voltage $u_{o}(t)$. As predicted from theoretical analysis in the previous section, the currents store to their desired equilibrium values $i_{d}^{*}=I_{d}$ and $I_{q}^{*}=0$, and the output capacitor voltage rapidly restores to its desired value $U_{o}$ without any overshoot due to the parallel damping resistor value defined in (16). Also notice that the input inductor currents are precisely in phase with the source voltages as shown in Figure 4. Hence, the control objective C.1-C.2 is achieved.

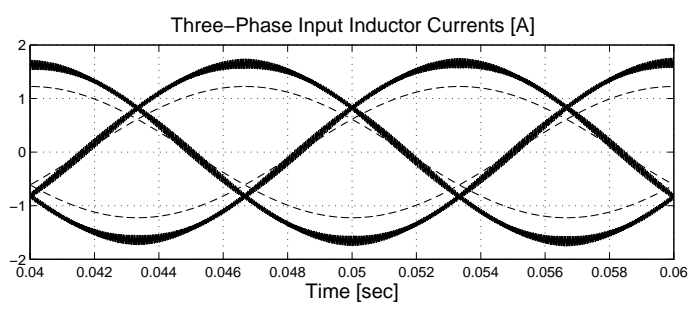

Fig. 4. AC-side current responses in steady state for $\Delta R_{o}=-\frac{1}{2} R_{o}$. The dashed lines represent the scaled three-phase source voltages.

\section{CONCLUSION AND OUTLOOK}

In this paper we have developed a $\mathrm{PBC}$ scheme for the three-phase AC/DC Boost rectifier that is robust against load perturbations. The key idea is to use the parallel damping injection scheme that has been proven to be robust for DC/DC converters. However, in this case, first a pre-compensation on the AC input stage should be done. This pre-compensation may give rise to another type of robustness problems, namely a closed-loop system that is not robust against variations of the input inductances. Nevertheless, it seems that this can be easily solved by adding an additional adaptive mechanism that compensates for these variations. This appears to be more practically appealing than the alternative of adding an adaptive mechanism for the load variations.

\section{REFERENCES}

Brayton, R.K. and J.K. Moser (1964). A theory of nonlinear networks I. Quart. Appl. Math. 12(1), 1-33.

Jeltsema, D. and J.M.A. Scherpen (2004). Tuning of passivity-preserving controllers for switchedmode power converters. IEEE Trans. Automatic Control 49, 1333-1344.

Kugi, A. (2001). Nonlinear Control Based on Physical Models. Springer-Verlag.

Lee, T.S. (2004). Lagrangian modeling and passivitybased control of three-phase AC/DC voltagesource converters. IEEE Trans. Indust. Electr. 51(4), 892-902.

Leonhard, W. (1985). Control of electrical drives. Elsevier.

Ortega, R., A. Loría, P.J. Nicklasson and H. SiraRamírez (1998). Passivity-Based Control of EulerLagrange Systems; Mechanical, Electrical and Electromechanical Applications. Springer-Verlag.

Scherpen, J.M.A., D. Jeltsema and J.B. Klaassens (2003). Lagrangian modeling of switching electrical networks. Systems and Control Letters 48(5), 365-374.

Sira-Ramírez, H., R.A. Perez-Moreno, R. Ortega and M. Garcia-Esteban (1997). Passivity-based controllers for the stabilization of dc-to-dc power converters. Automatica 33, 499-513.

Stern, T.E. (1965). Theory of Nonlinear Networks and Systems. Addison-Wesley, Reading, Massachusetts. 